\title{
van der Waals frictional drag induced by liquid flow in low-dimensional systems
}

\author{
A. I. Volokitin ${ }^{1,2, *}$ and B. N. J. Persson ${ }^{1}$ \\ ${ }^{1}$ Institut für Festkörperforschung, Forschungszentrum, D-52425 Jülich, Germany \\ ${ }^{2}$ Samara State Technical University, 443100 Samara, Russia
}

(Received 29 July 2007; published 22 January 2008)

\begin{abstract}
We study the van der Waals frictional drag force induced by liquid flow in low-dimensional systems [two-dimensional (2D) and one-dimensional (1D) electron systems, and 2D and 1D channels with liquid]. We find that for both $1 \mathrm{D}$ and 2D systems, the frictional drag force induced by liquid flow may be several orders of magnitude larger than the frictional drag induced by electronic current.
\end{abstract}

DOI: 10.1103/PhysRevB.77.033413

PACS number(s): 82.70. $-\mathrm{y}, 44.40 .+\mathrm{a}, 68.35 . \mathrm{Af}$

A great deal of attention has been devoted to the problem of frictional drag in low-dimensional systems ${ }^{1-7}$ because of it importance for nanoscale detectors. Such detectors would be of great interest in micromechanical and biological applications, ${ }^{8,9}$ where local dynamical effects are intensely studied.

A frictional drag will act on a two-dimensional (2D)electron system if an electric current flows in a second parallel 2D-electron system. This drag effect, suggested many years ago, ${ }^{10,11}$ has been studied in two-dimensional quantum wells. ${ }^{6,7}$ Experiments ${ }^{6}$ show that, at least for small separations, the frictional drag can be explained by the interaction between the electrons in the different layers via the fluctuating Coulomb field. However, for large interlayer separation the friction drag is dominated by phonon exchange. ${ }^{12}$

Recently, it was observed that the flow of a liquid over bundles of single-walled carbon nanotubes induces a voltage in the sample along the direction of the flow. ${ }^{1,2}$ The dependence of the voltage on the flow speed was found to be logarithmic over five decades of variation of the speed. There have been attempts to explain this flow-induced voltage in electrokinetic terms, as a result of the streaming potential that develops along the flow of an electrolyte through a microporous insulator. ${ }^{13,14}$ Earlier Král and Shapiro ${ }^{4}$ proposed that the liquid flow transfers momentum to the acoustic phonons of the nanotube, and that the resulting "phonon wind" drives an electric current in the nanotube. They also suggested, qualitatively, that the fluctuating Coulomb field of the ions in the liquid could drag directly the carriers in the nanotube. However, the first mechanism ${ }^{4}$ requires an enormous pressure, ${ }^{2}$ while the second mechanism ${ }^{4}$ results in a very small current, of order femtoamperes. ${ }^{2}$ In Ref. 2 another mechanism was proposed, which is related to the second idea of Ref. 4, but which requires neither localization of carriers nor drag at the same speed as the ions. In fact, Ref. 2 considered the friction between a moving point charge and the surrounding medium. An external charge will induce an "image" charge in the surrounding medium. Because of the finite response time this image charge lags behind the moving charge, which results in a force acting on the charge, referred to as the electrostatic friction. For neutral systems, such as a nanotube, the electrostatic friction proposed in Ref. 2 will vanish.

In Ref. 5 it was assumed that the liquid molecules nearest to the nanotube form a $2 \mathrm{D}$ solidlike monolayer, pinned to the nanotube by adsorbed ions. As the liquid flows, the adsorbed solid monolayer performs stick-slip type of sliding motion along the nanotube. The drifting adsorbed ions will produce a voltage in the nanotube through electronic friction against free electrons inside the nanotube.

In Ref. 3 model calculations of the frictional drag were presented involving a channel containing overdamped Brownian particles. The channel was embedded in a wide chamber containing the same type of Brownian particles with drift velocity parallel to the channel. It was found that the flow of particles in the chamber induces a drift of the particles in the channel.

In this Brief Report we will address the intriguing idea of using frictional drag as a noncontact means to detect motion in surrounding liquid. We present a frictional drag theory based on the theory of the van der Waals friction. ${ }^{15,17,16}$ The origin of the van der Waals friction is connected with the fluctuating electromagnetic field which is always present outside of any medium due to thermal and quantum fluctuations of the charge density inside the medium. This fluctuating electromagnetic field induces polarization of the medium, and is responsible for many important phenomena such as radiative heat transfer and the van der Waals interaction. ${ }^{16}$ When two media are in relative motion, the induced polarization will lag behind the fluctuating polarization inducing it, and this gives rise to the so-called van der Waals friction.

The origin of the van der Waals friction is closely connected with the Doppler effect. Let us consider two flat parallel surfaces, separated by a sufficiently wide insulator gap, which prevents particles from tunneling across it. If the charge carriers inside the volumes restricted by these surfaces are in relative motion (velocity $v$ ) a frictional stress will act between surfaces. This frictional stress is related

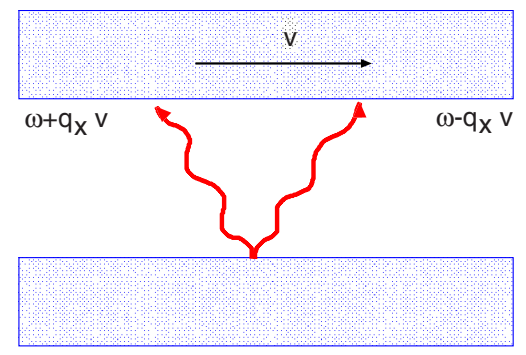

FIG. 1. (Color online) Two bodies moving relative to each other will experience van der Waals friction due to Doppler shift of the electromagnetic waves emitted by them. 
with an asymmetry of the reflection amplitude along the direction of motion; see Fig. 1. If one body emits radiation, then in the rest reference frame of the second body these waves are Doppler shifted which will result in different reflection amplitudes. The same is true for radiation emitted by the second body. The exchange of "Doppler-shifted photons" will result in momentum transfer and to the van der Waals friction. According to Refs. 15 and 17, the frictional stress between two flat parallel surfaces at separation $d \ll \lambda_{T}$ $=c \hbar / k_{B} T$ is determined by

$$
\begin{aligned}
\sigma_{\|}= & \frac{\hbar}{2 \pi^{3}} \int_{-\infty}^{\infty} d q_{y} \int_{0}^{\infty} d q_{x} q_{x} e^{-2 q d}\left\{\int_{0}^{\infty} d \omega\left[n(\omega)-n\left(\omega+q_{x} v\right)\right]\right. \\
& \times\left[\frac{\operatorname{Im} R_{1 p}\left(\omega+q_{x} v\right) \operatorname{Im} R_{2 p}(\omega)}{\left|1-e^{-2 q d} R_{1 p}\left(\omega+q_{x} v\right) R_{2 p}(\omega)\right|^{2}}+(1 \leftrightarrow 2)\right] \\
& -\int_{0}^{q_{x} v} d \omega[n(\omega)+1 / 2]\left[\frac{\operatorname{Im} R_{1 p}\left(\omega-q_{x} v\right) \operatorname{Im} R_{2 p}(\omega)}{\left|1-e^{-2 q d} R_{1 p}\left(\omega-q_{x} v\right) R_{2 p}(\omega)\right|^{2}}\right. \\
& +(1 \leftrightarrow 2)]\},
\end{aligned}
$$

where $n(\omega)=\left[\exp \left(\hbar \omega / k_{B} T\right)-1\right]^{-1}$ and $(1 \leftrightarrow 2)$ denotes the terms which are obtained from the preceding terms by permutation of indices 1 and 2. $R_{i p}(i=1,2)$ is the reflection amplitude for surface $i$ for $p$-polarized electromagnetic waves. The reflection amplitudes for a 2D-electron system are determined by ${ }^{17}$

$$
R_{i p}=\frac{\epsilon_{i p}-1}{\epsilon_{i p}+1},
$$

where $\epsilon_{i p}=4 \pi i q \sigma_{i}(\omega, q) / \omega \varepsilon+1, \sigma_{i}$ is the longitudinal conductivity of the layer $i$, and $\varepsilon$ is the dielectric constant of the surrounding dielectric. The longitudinal conductivity can be written in the form $\sigma_{l}(\omega, q)=-i \omega \chi_{l}(\omega, q) / q^{2}$, where $\chi_{l}$ is the finite lifetime generalization of the longitudinal Lindhard response function for 2D-electron gas. ${ }^{18,19}$ The friction force per unit charge in the layer is determined by $E=\sigma_{\|} / n_{s} e$, where $n_{s}$ is the 2D-electron concentration in the layer. For $v \ll v_{F}$, where $v_{F}$ is the Fermi velocity, the friction force depends linearly on velocity $v$. For $d=175 \AA$ at $T=3 \mathrm{~K}$, and with $n_{s}=1.5 \times 10^{15} \mathrm{~m}^{-2}$, the electron effective mass $m^{*}$ $=0.067 m_{e}, v_{F}=1.6 \times 10^{7} \mathrm{~cm} / \mathrm{s}$, the electron mean free path $l=v_{F} \tau=1.21 \times 10^{5} \AA$, and $\varepsilon=10$ (which corresponds to the condition of the experiment) ${ }^{6}$ we get $E=6.5 \times 10^{-6} v \mathrm{~V} / \mathrm{m}$, where the velocity $v$ is in $\mathrm{m} / \mathrm{s}$. For a current $200 \mathrm{nA}$ in a two-dimensional layer with the width $w=20 \mu \mathrm{m}$ the drift of electrons (drift velocity $v=60 \mathrm{~m} / \mathrm{s}$ ) creates a frictional drag force per unit charge in the adjacent quantum well $E=4$ $\times 10^{-4} \mathrm{~V} / \mathrm{m}$. Note that for the electron systems the frictional drag force decreases when the electron concentration increases. For a example, for 2D-quantum wells with high electron density $\left(n_{s}=10^{19} \mathrm{~m}^{-2}, T=273 \mathrm{~K}, \tau=4 \times 10^{-14} \mathrm{~s}, \varepsilon\right.$ $=10$, and $m^{*}=m_{e}$ ) at $d=175 \AA$ we get $E=1.2 \times 10^{-9} \mathrm{v} \mathrm{V} / \mathrm{m}$.

Let us replace one $2 \mathrm{D}$ layer by a semi-infinite chamber with liquid containing ions. The reflection amplitude for the interface between dielectric and liquid is given by Eq. (2), where

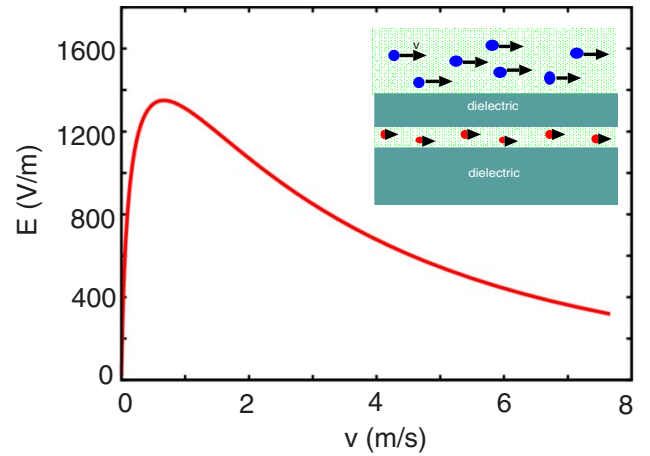

FIG. 2. (Color online) The frictional drag force per unit charge for ions in a $2 \mathrm{D}$ channel with liquid induced by liquid flow in a semi-infinite chamber as a function of the flow velocity for identical liquids in the channel and chamber. The temperature $T=300 \mathrm{~K}$, the ion concentration in liquid $n=10^{24} \mathrm{~m}^{-3}$, the diffusion coefficients of ions $D=10^{-9} \mathrm{~m}^{2} / \mathrm{s}$, the dielectric constant of the liquid $\varepsilon_{0}=80$, and the separation between the channel and semi-infinite chamber $d$ $=1 \mathrm{~nm}$.

$$
\epsilon=\frac{\varepsilon_{0} \lambda\left(D q_{D}^{2}-i \omega\right)}{\varepsilon\left(D q_{D}^{2} q-i \omega \lambda\right)}
$$

where $D$ is the diffusion coefficient of the ions, $\varepsilon_{0}$ is the dielectric function of liquid without ions, $q_{D}$ $=\sqrt{4 \pi n e^{2} / \varepsilon_{0} k_{B} T}$ is the Debye screening wave number, $\lambda$ $=\sqrt{q^{2}+q_{D}^{2}-i \omega / D}$, and $n$ is the ion concentration. In this case, for $v \ll v_{F}$ the friction force also depends linearly on velocity $v$. In particular, for $n=10^{24} \mathrm{~m}^{-3}, T=273 \mathrm{~K}, \varepsilon_{0}=80$, and $D$ $=10^{-9} \mathrm{~m}^{2} / \mathrm{s}$ for high electron density $\left(n_{s}=10^{19} \mathrm{~m}^{-2}\right)$ for 2Delectron system we get $E=1.4 \times 10^{-6} v \mathrm{~V} / \mathrm{m}$. This friction is 3 orders of magnitude larger than the friction between the 2D-electron systems with high electron concentration, and of the same order of magnitude as the friction between 2Delectron systems with low electron concentration.

Let us replace the second 2D layer by a narrow 2D channel with thickness $d_{c}$. For $q_{D} d_{c} \ll 1$ the flowing liquid in the channel can be considered as a $2 \mathrm{D}$ liquid. The conductivity of a $2 \mathrm{D}$ liquid with ions performing Brownian motion is given by

$$
\sigma(\omega, q)=-\frac{i \omega d_{c}}{4 \pi}\left[-1+\varepsilon_{c}\left(1+\frac{q_{D c}^{2}}{q^{2}-i \omega / D_{c}}\right)\right],
$$

where $\varepsilon_{c}, D_{c}$, and $q_{D c}$ are the dielectric function, the diffusion coefficient, and the Debye wave number for the liquid in the channel, respectively. Figure 2 shows the dependence of the frictional drag force per unit charge in the channel on the liquid flow velocity in the semi-infinite chamber for identical liquid in the channel and in the chamber. We have used the same parameters as used above for the liquid and with the separation between the channel and chamber $d=1 \mathrm{~nm}$. The frictional drag force on the ions in the channel initially increases with the flow velocity, reaches a maximum, and then decreases at large value of the flow velocity, in agreement with the model calculation in Ref. 3. The position of maximum decreases when the density of ions decreases. The frictional drag force induced by liquid flow in the narrow chan- 
nel is 9 orders of magnitude larger than the friction force induced in a 2D-electron system.

As a limiting case of the situation considered above, let us consider a 2D system immersed in a flowing liquid in an infinite chamber. We assume that the liquid flows along the $x$ axis, and that the plane of 2D system coincides with the $x y$ plane. According to the fluctuation-dissipation theorem, the average value of the correlation function for the Fourier components of the Coulombic potential for a infinite medium in the plane of $2 \mathrm{D}$ system is determined by ${ }^{16}$

$$
\left\langle\varphi^{f}(\omega, q, 0) \varphi^{* f}(\omega, q, 0)\right\rangle=4 \hbar[n(\omega)+1 / 2] \operatorname{Im} \Sigma(\omega, q, 0),
$$

where

$$
\Sigma(\omega, q, 0),=-\int_{-\infty}^{\infty} \frac{d k_{z}}{2 \pi} \frac{1}{k^{2} \varepsilon_{0} \varepsilon(k)},
$$

where $\mathbf{k}=\left(\mathbf{q}, k_{z}\right)$. The dielectric function of the Debye plasma is determined by

$$
\varepsilon(k)=1+\frac{q_{D}^{2}}{k^{2}-i \omega / D}
$$

According to the fluctuation-dissipation theorem the average value of the correlation function for the Fourier components of the fluctuating current density in 2D system is determined by ${ }^{16}$

$$
\left\langle j_{q}^{f}(\omega, q) j_{q}^{f^{*}}(\omega, q)\right\rangle=\frac{\hbar q^{2}}{\pi \omega}[n(\omega)+1 / 2] \operatorname{Re} \sigma(\omega, q) .
$$

The friction force per unit area of 2D system is given by

$$
\sigma_{\|}=\int \frac{d^{2} \mathbf{q}}{(2 \pi)^{2}} \frac{q_{x}}{q}\left\langle E_{q}(\omega, q, 0) \rho^{*}(\omega, q)\right\rangle
$$

where $E_{q}=-i q \varphi_{q}=E_{q}^{f}+E_{q}^{i n d}, \rho=\rho^{f}+\rho^{i n d}$. The fluctuating electric field $E_{q}^{f}$ is the sum of the fluctuating field which exists in the infinite medium without the 2D system, and the electric field created by the fluctuating charge density $\rho_{q}^{f}=q j_{q}^{f} / \omega$ in the presence of the flowing liquid. The induced electric field $E_{q}^{\text {ind }}$ is created by the charge density $\rho_{q}^{\text {ind }}=q j_{q}^{\text {ind }} / \omega$ induced in the 2D system by the electric field $E_{q}$. The correlation functions (5) and (8) are determined in the rest reference frames of the liquid, and 2D system, respectively. To find the relation between electric fields in the different reference frame we use the Galilean transformation, which will give rise to Doppler shift of the frequencies of the electric fields in the different reference frames. Solving the Poisson's equation with the fluctuating charge density $\rho^{f}$, and the charge density induced by the fluctuating electric field $E_{q}^{f}=-i q \varphi^{f}$ as the sources of the field we get $E_{q}$, and from Ohm law we get $\rho$. The result of these calculations is

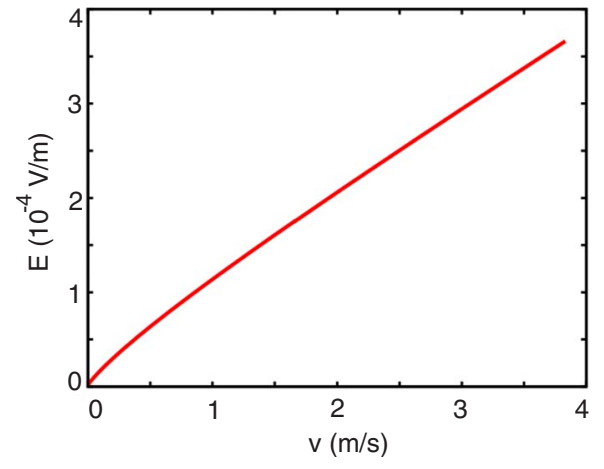

FIG. 3. (Color online) The frictional drag force per unit charge in a 1D-electron system induced by liquid flow in infinite chamber as a function of the flow velocity with the same parameters for liquid as for Fig. 2. The electron concentration per unit length in 1D system $n_{l}=3 \times 10^{9} \mathrm{~m}^{-1}$, and the electron relaxation time $\tau=4$ $\times 10^{-14} \mathrm{~s}$.

$$
\begin{aligned}
\sigma_{\|}= & \frac{\hbar}{2 \pi^{2}} \int_{-\infty}^{\infty} d q_{y} \int_{0}^{\infty} d q_{x} q_{x} q^{2}\left\{\int_{0}^{\infty} d \omega\left[n(\omega)-n\left(\omega+q_{x} v\right)\right]\right. \\
& \times\left[\frac{\operatorname{Re} \sigma\left(\omega+q_{x} v\right) \operatorname{Im} \Sigma(\omega)}{\left(\omega+q_{x} v\right)\left|1-4 \pi i q^{2} \sigma\left(\omega+q_{x} v\right) \Sigma(\omega) /\left(\omega+q_{x} v\right)\right|^{2}}\right. \\
& \left.+\left(\omega+q_{x} v \leftrightarrow \omega\right)\right]-\int_{0}^{q_{x} v} d \omega[n(\omega)+1 / 2] \\
& \times\left[\frac{\operatorname{Re} \sigma\left(\omega-q_{x} v\right) \operatorname{Im} \Sigma(\omega)}{\left(\omega-q_{x} v\right)\left|1-4 \pi i q^{2} \sigma\left(\omega-q_{x} v\right) \Sigma(\omega) /\left(\omega-q_{x} v\right)\right|^{2}}\right. \\
& \left.\left.+\left(\omega-q_{x} v \leftrightarrow \omega\right)\right]\right\},
\end{aligned}
$$

where $\left(\omega \pm q_{x} v \leftrightarrow \omega\right)$ denote the terms which are obtained from the preceding terms by permutations of the arguments $\omega \pm q_{x} v$ and $\omega$. With the same parameters as used above for the liquid, and for the high density 2D-electron system, we get $E=8.1 \times 10^{-6} v \mathrm{~V} / \mathrm{m}$. For a $1 \mathrm{D}$-electron system we obtained a formula which is similar to Eq. (10). Figure 3 shows the result of the calculations of the friction drag force (per unit charge) for a $1 \mathrm{D}$-electron system with the electron density per unit length $n_{l}=3 \times 10^{9} \mathrm{~m}^{-1}$, the temperature $T$ $=300 \mathrm{~K}$, and with the same parameters for the liquid as used above. For the 1D-electron system we obtained a slight deviation from the linear dependence of the frictional drag on the liquid flow velocity. The frictional drag for the 1Delectron system is 1 order of magnitude larger than for the 2D-electron system.

Figure 4 shows the dependence of the frictional drag force per unit charge acting on the ions in the liquid in the $2 \mathrm{D}$ channel with liquid on the liquid flow velocity in the infinite chamber, assuming identical liquid in the channel and in the chamber. In this case the maximum in the frictional drag force is larger, and the decay at large velocities is slower in comparison with the semi-infinite chamber at separation $d$ $=1 \mathrm{~nm}$. Qualitatively, we obtained the same results for a $1 \mathrm{D}$ channel.

For a channel with open ends the frictional drag force will 


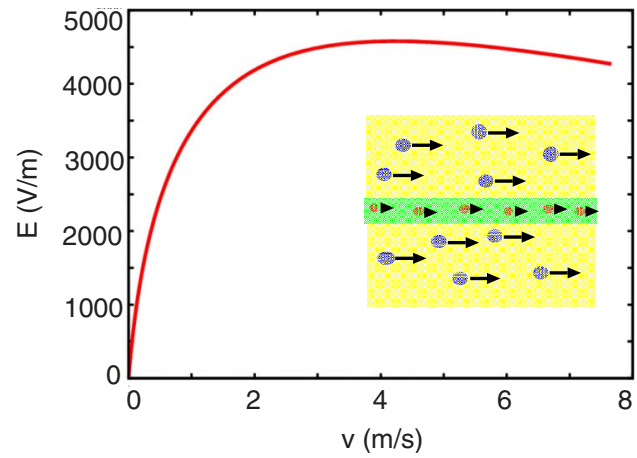

FIG. 4. (Color online) The same as Fig. 2 but for infinite chamber.

induce a drift motion of the ions in the liquid with velocity $v_{d}=D_{c} e E / k_{B} T$. The positive and negative ions will drift in the same direction. In contrast to electron systems, for the channel with closed ends, in the case of equal concentration of the positive and negative ions, the frictional drag will not induce a voltage because displacement of the ions under the action of the frictional drag force will not generate any nonzero macroscopic charge distribution in the liquid. However, the frictional drag will induce a pressure difference $\Delta p$ $=n L e E$, where $L$ is the length of the channel. For example, if $n=10^{24} \mathrm{~m}^{-3}, L=100 \mu \mathrm{m}$, and $E=1000 \mathrm{~V} / \mathrm{m}$ we get pressure difference $\Delta p=10^{4} \mathrm{~Pa}$, which should be easy to measure. Another situation realizes, when as a result of adsorption, one type of ions is fixed on the walls of channel, and the equal number of mobile ions of opposite sign is distributed in the liquid phase. In such system the motion of polar liquid in the adjacent region will lead to the frictional drag force acting on the mobile ions in the channel. In the channel with the closed ends this frictional drag will induce the voltage, which can be measured.

In this Brief Report we have shown that the van der Waals frictional drag force induced in low-dimensional system by liquid flow can be several orders of magnitude larger than the friction induced by electron current. For a narrow 2D and 1D channel with liquid the frictional drag force is several orders of magnitude larger than for 2D- and 1D-electron systems. In the contrast to electron system the frictional drag force for a narrow channel with liquid depends nonlinearly on the flow velocity. These results contradict to the calculations in Ref. 2 , where it was assumed that the observed nonlinear dependence of a voltage on the liquid flow velocity was connected with the frictional drag of electrons in the nanotubes. However, according to our calculations, frictional drag force due to van der Waals friction acts considerably greater on the mobile ions in the channels of porous medium than on the electrons in low-dimensional electronic structures. Thus, liquid flow-induced voltage observed in Ref. 2 is more probably connected with the frictional drag of mobile ions in an electrolyte in the channels between nanotubes, but not electrons in the nanotubes. In contrast to the streaming potential, ${ }^{2}$ which is created by liquid flow in the porous medium, the considered mechanism is connected with the liquid flow out of the porous medium; therefore it does not require an enormous pressure difference. These results should have a broad application for studying of the van der Waals friction and in the design of nanosensors.

A.I.V. acknowledges financial support from the Russian Foundation for Basic Research (Grant No. N 06-02-16979a), DFG, and the European Union Nanotribology project.
*Author to whom correspondence should be addressed. avodi@samgtu.ru

${ }^{1}$ S. Ghosh, A. K. Sood, and N. Kumar, Science 299, 1042 (2003).

${ }^{2}$ S. Ghosh, A. K. Sood, S. Ramaswamy, and N. Kumar, Phys. Rev. B 70, 205423 (2004).

${ }^{3}$ M. Das, S. Ramaswamy, A. K. Sood, and G. Ananthakrishna, Phys. Rev. E 73, 061409 (2006).

${ }^{4}$ P. Král and M. Shapiro, Phys. Rev. Lett. 86, 131 (2001).

${ }^{5}$ B. N. J. Persson, U. Tartaglino, E. Tosatti, and H. Ueba, Phys. Rev. B 69, 235410 (2004).

${ }^{6}$ T. J. Gramila, J. P. Eisenstein, A. H. MacDonald, L. N. Pfeiffer, and K. W. West, Phys. Rev. Lett. 66, 1216 (1991).

${ }^{7}$ U. Sivan, P. M. Solomon, and H. Shtrikman, Phys. Rev. Lett. 68, 1196 (1992).

${ }^{8}$ R. B. M. Schasfoort, S. Schlautmann, J. Hendrikse, and A. van der Berg, Science 286, 942 (1999).

${ }^{9}$ N. J. Munro, K. Snow, J. A. Kant, and J. P. Landers, Clin. Chem.
45, 1906 (1999).

${ }^{10}$ M. B. Pogrebinskii, Fiz. Tekh. Poluprovodn. (S.-Peterburg) 11,

637 (1977) [Sov. Phys. Semicond. 11, 372 (1977)].

${ }^{11}$ P. J. Price, Physica B \& C 117, 750 (1983).

${ }^{12}$ M. C. Bønsager, K. Flensberg, Ben Yu-Kuang Hu, and A. H. MacDonald, Phys. Rev. B 57, 7085 (1998).

${ }^{13}$ A. E. Cohen, Science 300, 1235 (2003).

${ }^{14}$ S. Ghosh, A. K. Sood, and N. Kumar, Science 300, 1235 (2003).

${ }^{15}$ A. I. Volokitin and B. N. J. Persson, J. Phys.: Condens. Matter 11, 345 (1999); Phys. Low-Dimens. Struct. 7/8, 17 (1998).

${ }^{16}$ A. I. Volokitin and B. N. J. Persson, Rev. Mod. Phys. 79, 1291 (2007).

${ }^{17}$ A. I. Volokitin and B. N. J. Persson, J. Phys.: Condens. Matter 13, 859 (2001).

${ }^{18}$ N. D. Mermin, Phys. Rev. B 1, 2362 (1970).

${ }^{19}$ F. Stern, Phys. Rev. Lett. 18, 546 (1967). 